http://dx.doi.org/10.15407/ukrbotj72.05.468

D.M. IAKUSHENKO ${ }^{1}$, O.O. ORLOV ${ }^{2}$

${ }^{1}$ University of Zielona Góra, Department of Biological Sciences

Z. Szafrana 1, Zielona Góra, 65-516, Poland

d.iakushenko@wnb.uz.zgora.pl

${ }^{2}$ G.M. Vysotsky Polisskiy Branch of URIFFM

Neskorenykh str., 2, Dovzhyk village, Zhytomyr District, Zhytomyr Region, 10004, Ukraine

orlov.botany@gmail.com

\title{
NEW RECORDS OF UTRICULARIA AUSTRALIS (LENTIBULARIACEAE) IN UKRAINE
}

Iakushenko D.M., Orlov O.O. New records of Utricularia australis (Lentibulariaceae) in Ukraine. - Ukr. Bot. J. - 2015. - 72(5): 468-473.

The data about the first findings of Utricularia australis in Ukrainian Polissia (Zhytomyr Region) are given. Ecological and coenotic patterns of this species for the new locations are presented. Species localities were represented by small artificial reservoirs in clay, peat and granite quarries, as well as ponds. It was revealed that Utricularia australis grew in four floristic associations: Utricularietum australis Müller et Görs 1960, Nitelletum mucronatae Corillion et Guerlesquin 1972, Equiseto fluviatilis-Caricetum rostratae Zumpfe 1929, Equisetetum fluviatilis Nowiński 1930. For the first time in Ukraine, the association Utricularietum australis is reported and its characteristic is given.

K e y w o r d s: Utricularia australis, aquatic vegetation, flora, Ukraine

\section{Introduction}

Utricularia australis R.Br. is a perennial free-floating submerged carnivorous aquatic plant species. In Europe the species occurs in temperate, boreal and mediterranean regions (Uotila, 2013). U. australis is listed in the Red Data Book of Ukraine (Chervona knyga..., 2009) as a vulnerable species. In Ukraine the species is known since 1997 from Transcarpathia (Prots', 2009); it was also registered in the Cis-Carpathian area in 2006 (Danylyk et al., 2007). A former locality in Dubliany, near Lviv (after Knapp, as U. neglecta Lehm.) listed in the «Flora of the Ukrainian SSR» (Visiulina, 1961) has not been recently confirmed.

Despite collections of $U$. australis made by $\mathrm{W}$. Żukowski in eastern Poland, in Polesie Lubelskie (Żukowski, 1974), this species has never been reported in botanical literature for the neighboring area of Ukrainian Polissia.

During the study of the flora and vegetation in Zhytomyr Region (northern Ukraine - Ukrainian Polissia), the authors discovered several new localities of $U$. australis. These new points significantly extend the knowledge about the species distribution in Ukraine. The aim of the paper is to inform about these new records and to describe briefly the ecological-coenotic patterns of this species in the studied area.

(C) D.M. IAKUSHENKO, O.O. ORLOV, 2015

\section{Methods}

Field works were conducted in 2012 and 2014. Identification of $U$. australis was carried out according to the identification keys of W. Żukowski (1970), P. Taylor (1972) and Š. Husák (2000). Plant communities were studied according to J. Braun-Blanquet approach (Westhoff \& van der Maarel, 1973), phytosociological relevés were stored using TURBOVEG software (Hennekens \& Schaminée, 2001). Phytosociological nomenclature was given after K. Šumberová (2011), K. Šumberová et al. (2011) and D. Iakushenko \& O. Borysova (2012). The Ellenberg indicator values were calculated using JUICE 7.0 software (Tichý, 2002).

\section{Results}

In July 2012, a small population of $U$. australis was found by $D$. Iakushenko in the southern vicinities of Korostyshiv town. In July-August 2014, four new localities of the species were observed in Ukrainian Polissia by O. Orlov.

The list of the localities is given below (Fig. 1):

1. Zhytomyr Region, Korostyshiv District, S vicinities of Korostyshiv town, Kyrychanka (N 50 17'44.95" E 2903'36.67”); pond; 19.07.2012; D. Iakushenko;

2. Zhytomyr Region, Zhytomyr District, $0.5 \mathrm{~km} \mathrm{~N}$ Barashivka village (N 50²8'58.70” E 28 54'25.87”); clay quarry; 06.07.2014, 25.08.2014; O. Orlov (Fig. 2); 


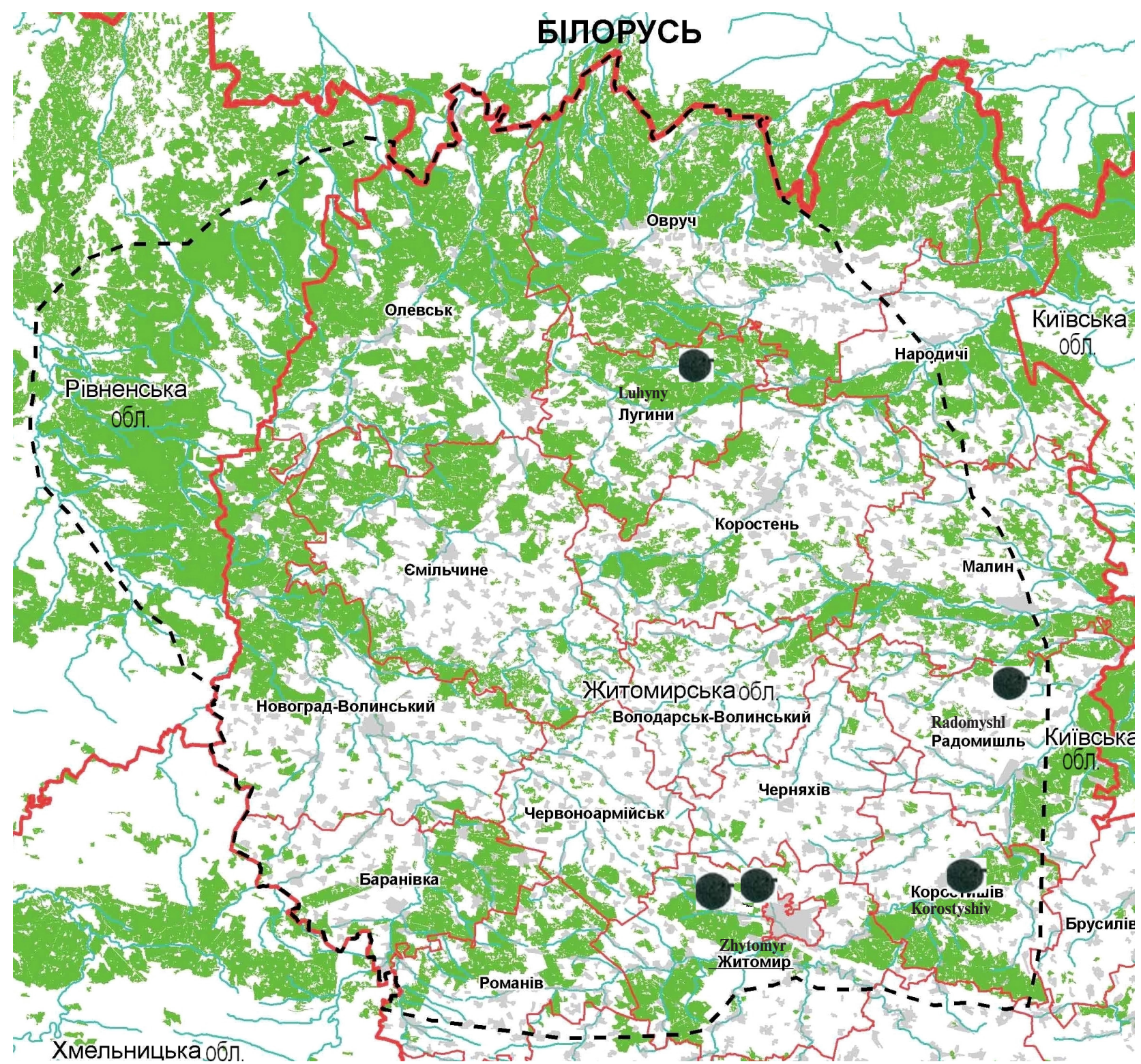

Fig. 1. Localities of Utricularia australis in Ukrainian Polissia (dotted line restricts Zhytomyr Polissia, the central part of the Region)

Fig. 2. Utricularia australis in a clay quarry near Barashivka village

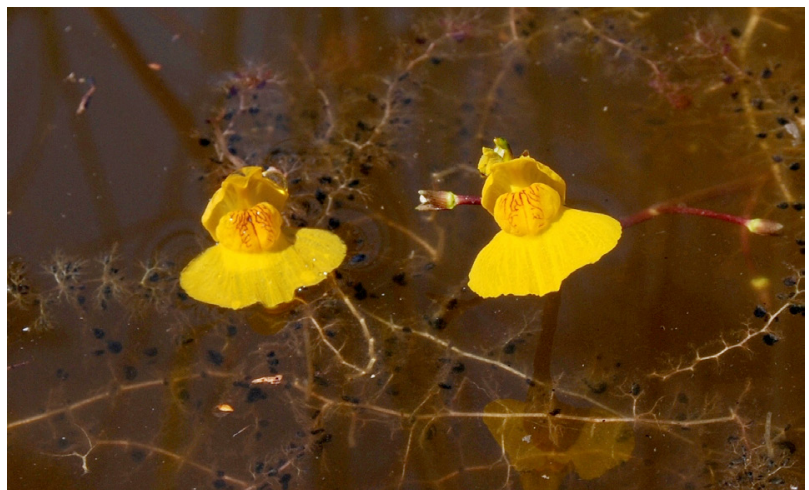


3. Zhytomyr Region, W vicinities of Zhytomyr city, Bohunia (N50²9'03.53" E 2860'66.81"); ephemeral water body near granite quarry; 27.07.2014; O. Orlov; 4. Zhytomyr Region, Luhyny District, Lypnyky village (N 51¹8'34.69" E 2844'63.63"); pond; 05.08.2014; O. Orlov;

5. Zhytomyr Region, Radomyshl District, $2 \mathrm{~km} \mathrm{SW}$ Fedorivka village (N 5041'45.73” E 29²2'26.08”); peat pit; 16.08.2014; O. Orlov.

Scarce phytosociological material regarding $U$. australis from Ukraine was published so far. Only 3 relevés were published from Transcarpathia (vicinities of Dyida settlement, Berehovo District) (Drescher, Prots', Mountford, 2003): 2 relevés belong to the association Stratiotetum aloidis Miljan 1933 and one relevé was made in community of Butometum umbellati Philippi 1973. In the area of National Nature Park «Hutsulshchyna» (Ivano-Frankivsk Region) $U$. australis was noted in one locality as synusia in communities of Phragmitetum communis Savič 1926 and Typhetum angustifoliae Pignatti 1953 (Danylyk et al., 2007; Iakushenko et al., 2011): P. 275, Table 9.15, relevés $1-2$ made by L. Borsukevych. In the Red Data Book of Ukraine it is mentioned that this species forms the community Lemno-Utricularietum australis and also occurs in some other associations of Lemnetea class (Prots', 2009).

In the study region, $U$. australis forms freefloating pleustonic communities typical for aquatic bladderworts; however, it also occurs as a synusial aggregation in the complex of the littoral vegetation. Four associations were distinguished according to our data (Table).

Utricularietum australis Müller et Görs 1960 is a focal community in the study (Table, rel. 1-8). These plant communities were observed in shallow water bodies on depths from 0.15 to $0.90 \mathrm{~m}$, with sandy, peat, or clay bottom covered by muddy sediments. The cover of $U$. australis changed from 30 to $80 \%$. The total cover varied from $50-60 \%$ to $95-100 \%$, and the cover of natant layer - from 15 to $50 \%$. The number of species among coenoses differed significantly - from 1 (i.e. monodominant patches) to $10-12$ vascular plant species per relevé. The second constant species was Lemna minor L.; other pleustonic species (Hydrocharis morsusranae L. and Lemna trisulca L.) were less frequent.

According to published phytosociological materials (Dubyna, 2006; Solomakha, 2008), this association has not yet been reported in Ukraine. Thus, association characteristic was done in the form acceptable for the Prodromus of Vegetation of Ukraine.

\section{Syntaxonomical position:}

Lemnetea O. de Bolòs et Masclans 1955

Lemnetalia minoris O. de Bolòs et Masclans 1955

Utricularion vulgaris Passarge 1964

\section{Association Utricularietum australis Müller et Görs} 1960

Synonims: Lemno-Utricularietum neglectae (Müller et Görs 1960) Passarge 1978, Potamo-Utricularietum australis (Müller et Görs 1960) Passarge 1996, Utricularietum neglectae Müller et Görs 1960.

Diagnostic species: Utricularia australis (opt., dom.)

Habitats: mesotrophic and eutrophic standing or slow-flowing, from slightly acid to neutral waters (shallow water bodies: small ponds, forest pools, open pits, etc.) with different types of bottom sediments.

\section{Ecological requirements:}

Mean of Ellenberg indication values (based on rel. $2-5,7-8$, Table): reaction $-6.59 \pm 0.68$; light $7.01 \pm 0.21$; continentality $-3.85 \pm 0.48$; nutrients $6.08 \pm 0.54$; moisture - $10.41 \pm 0.67$; temperature $5.52 \pm 0.22$.

Distribution in Ukraine: Zhytomyr Region. Patches of $U$. australis are also recorded for Transcarpathian and Ivano-Frankivsk Regions.

General distribution: Austria (Schratt, 1993), Czech Republic (Šumberová, 2011), Denmark (Lawesson, 2004), France (Felzines, 2012), Germany (Pott, 1995), Italy (Lastrucci et al., 2014), Lithuania (Sinkevičienè, 2013), Poland (Spałek, 2006), Portugal (Costa et al., 2012), Slovakia (Hrivnák, 2002), Spain (RivasMartínezet al., 2001). The association was described from SW Germany (Müller \& Görs, 1960).

Protected status: The diagnostic species (U. australis) is listed in the Red Data Book of Ukraine (Prots', 2009). The association should be protected on the national level and listed in the new edition of the Green Data Book of Ukraine.

Besides pleustonic communities, the patches with domination of $U$. australis in the region were found in coenoses of the associations Nitelletum mucronatae Corillion et Guerlesquin 1972 (Table, rel. 9), Equiseto fluviatilis-Caricetum rostratae Zumpfe 1929 (syn. Carietum rostratae Rübel 1912) (Table, rel. 10), and Equisetetum fluviatilis Nowiński 1930 (Table, rel. 11).

A phytosociological survey of $U$. australis in Ukraine should be a purpose of further study. 
Phytosociological characteristic of Utricularia australis in the studied localities

\begin{tabular}{|c|c|c|c|c|c|c|c|c|c|c|c|c|}
\hline Relevé number & 1 & 2 & 3 & 4 & 5 & 6 & 7 & 8 & \multirow{7}{*}{ 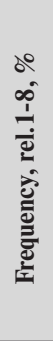 } & 9 & 10 & 11 \\
\hline $\begin{array}{l}\text { Locality } \\
\text { (according to numbers listed in the text) }\end{array}$ & 4 & 1 & 3 & 3 & 3 & 5 & 3 & 3 & & 2 & 4 & 4 \\
\hline Relevé area, m² & 12 & 4 & 4 & 6 & 4 & 10 & 4 & 4 & & 4 & 20 & 25 \\
\hline Number of species & 1 & 8 & 8 & 12 & 10 & 8 & 7 & 6 & & 15 & 10 & 7 \\
\hline Depth, m & 0.9 & 0.5 & 0.3 & 0.2 & 0.2 & 0.9 & 0.2 & 0.2 & & 0.4 & 0.8 & 0.7 \\
\hline Total cover, $\%$ & 50 & 95 & 85 & 90 & 95 & 90 & 60 & 100 & & 100 & 95 & 85 \\
\hline Syntaxon number & \multicolumn{8}{|c|}{1} & & 2 & 3 & 4 \\
\hline \multicolumn{13}{|l|}{ D.s. Ass. Utricularietum australis } \\
\hline Utricularia australis & 5 & 5 & 3 & 3 & 4 & 5 & 4 & 4 & 100 & 5 & 5 & 4 \\
\hline \multicolumn{13}{|l|}{ D.s. Ass. Nitelletum mucronatae } \\
\hline Nitella mucronata & . & . & . & . & . & . & . & . & . & 5 & . & $\cdot$ \\
\hline \multicolumn{13}{|c|}{ D.s. Ass. Equiseto fluviatilis-Caricetum rostratae } \\
\hline Carex rostrata & $\cdot$ & . & . & . & . & . & $\cdot$ & . & . & $\cdot$ & 4 & $\cdot$ \\
\hline \multicolumn{13}{|l|}{ D.s. Ass. Equisetetum fluviatilis } \\
\hline Equisetum limosum & . & . & . & . & $\cdot$ & $\cdot$ & $\cdot$ & . & . & $\cdot$ & . & 4 \\
\hline \multicolumn{13}{|l|}{ D.s. Cl. Lemnetea } \\
\hline Lemna minor & . & 1 & + & 1 & 1 & 1 & . & 2 & 75 & 1 & . & $\cdot$ \\
\hline Hydrocharis morsus-ranae & . & 2 & . & 1 & . & + & 2 & 2 & 63 & 1 & . & . \\
\hline Lemna trisulca & . & 1 & 2 & 2 & 2 & . & $\cdot$ & . & 50 & $\cdot$ & . & $\cdot$ \\
\hline Lemna gibba & . & . & . & . & + & . & . & . & 13 & . & . & . \\
\hline Spirodella polyrhiza & . & . & . & . & . & + & . & . & 13 & . & . & $\cdot$ \\
\hline \multicolumn{13}{|l|}{ D.s. Cl. Potametea } \\
\hline Elodea canadensis & . & 1 & 1 & . & 1 & 1 & . & . & 50 & 1 & . & $\cdot$ \\
\hline Batrachium circinatum & . & . & . & . & 2 & . & 1 & $\cdot$ & 25 & 2 & . & $\cdot$ \\
\hline Potamogeton natans & . & . & $\cdot$ & 1 & 2 & . & . & . & 25 & . & . & $\cdot$ \\
\hline \multicolumn{13}{|l|}{ D.s. Cl. Charetea } \\
\hline Chara globularis & $\cdot$ & . & 1 & 2 & . & 1 & $\cdot$ & . & 38 & $\cdot$ & $\cdot$ & $\cdot$ \\
\hline \multicolumn{13}{|l|}{ D.s. Cl. Phragmito-Magno-Caricetea } \\
\hline Alisma plantago-aquatica & $\cdot$ & . & 1 & 1 & 1 & . & . & . & 38 & 2 & + & $\cdot$ \\
\hline Eleocharis palustris & . & . & 1 & . & . & . & 2 & . & 25 & . & . & . \\
\hline Rumex hydrolapathum & . & 1 & . & + & . & . & . & . & 25 & . & . & . \\
\hline Carex acutiformis & . & + & . & . & . & . & . & 1 & 25 & + & . & + \\
\hline Glyceria fluitans & . & . & . & 1 & . & . & . & 2 & 25 & . & 1 & + \\
\hline Lythrum salicaria & . & . & . & 1 & . & . & . & . & 13 & . & + & + \\
\hline Typha latifolia & . & . & . & . & . & 1 & . & . & $\cdot$ & . & . & 1 \\
\hline Lycopus europaeus & . & . & . & . & . & . & . & . & $\cdot$ & + & + & $\cdot$ \\
\hline \multicolumn{13}{|l|}{ Other species: } \\
\hline Juncus conglomeratus & . & . & . & . & . & . & 1 & . & 13 & $\cdot$ & + & $\cdot$ \\
\hline Ranunculus flammula & $\cdot$ & . & . & . & . & . & . & 1 & 13 & + & . & $\cdot$ \\
\hline
\end{tabular}

Notes. Species noted in one relevé only: rel. 2 - Sparganium emersum (+); rel. 3 - Potamogeton gramineus (2); rel. 4 - Epilobium hirsutum (1), Lysimachia vulgaris (1); rel. 5 - Juncus articulatus (2), Myriophyllum spicatum (1); rel. 6 - Typha angustifolia (2); rel. 7 - Phragmites australis (2), Rorippa amphibia (1); rel. 9 - Bidens cernua (1), B. frondosa (1), Callitriche stagnalis (1), Glyceria plicata (2), Potamogeton trichoides (1); rel. 10 - Naumburgia thyrsiflora (1), Poa palustris (+), Potamogeton berchtoldii (1); rel. 11 - Hottonia palustris (1).

Syntaxon numbers: 1 - Utricularietum australis Müller et Görs 1960, 2 - Nitelletum mucronatae Corillion et Guerlesquin 1972, 3 - Equiseto fluviatilis-Caricetum rostratae Zumpfe 1929, 4 - Equisetetum fluviatilis Nowiński 1930. 


\section{Conclusion}

Five new localities of the protected aquatic carnivorous plant species, Utricularia australis, were reported for Zhytomyr Region. For the first time in Ukraine, the association of Utricularietum australis was characterized based on phytosociological relevés. The authors believe that $U$. australis belongs to so called "neglected" species of the Ukrainian flora (its synonymous name, $U$. neglecta, seems to be remarkably appropriate). Undoubtedly, this species is rare but more widely distributed in Ukraine than it was estimated earlier. It is supposed that new records of $U$. australis will be found on the whole territory of Ukraine as there are no climatic, ecological and coenotic limitations for this species in the country. More accurate floristic research in Ukraine is still needed.

Samples of $U$. australis have been transferred to the National Herbarium $(K W)$ of the M.G. Kholodny Institute of Botany, NAS of Ukraine.

Authors thanks cordially Dr. Andreas Fleischmann (Ludwig-Maximilians-Universität, München, Germany), Prof. Waldemar Żukowski (Adam Mickiewicz University, Poznań, Poland), and Dr. Liubov Borsukevych (Ivan Franko National University, Lviv, Ukraine) for confirmation of specimen identifications.

\section{REFERENCES}

Red Data Book of Ukraine. Vegetable Kingdom. Ed. Ia.P. Didukh, Kyiv: Globalkonsalting, 2009, 912 p. [Червона книга України: рослинний світ / За ред. Я.П. Дідуха. К.: Глобалконсалтинг, 2009. - 912 с.].

Costa J.C., Neto C., Aguiar C., Capelo J., Espírito Santo M.D., Honrado J., Pinto-Gomes C., Monteiro-Henriques T., Sequeira M., Lousã M. Vascular plant communities in Portugal (Continental, Azores and Madeira). In: Global Geobotany. International J. Geobotanical Sci., 2012, 2, pp. 1-180.

Danylyk I.M., Solomakha V.A., Solomakha T.D., Tsymbaliuk Z.M., Ukr. Bot. J., 2007, 64(2), pp. 242-246 [Данилик I.M., Соломаха В.А., Соломаха Т.Д., Цимбалюк 3.M. Utricularia australis R.Br. (Lentibulariaceae) новий вид для флори Прикарпаття // Укр. ботан. журн. - 2007. - 64(2). - C. 242-246].

Drescher A., Prots B., Mountford O. The world of old oxbowlakes, ancient riverine forests and drained mires in the Tisza river basin (International excursion to Eastern Hungary and Transcarpathia, Ukraine 31.08.200204.09.2002), Fritschiana (Graz), 2003, 45, pp. 43-69.

Dubyna D.V. Vyshcha vodna roslynnist'. Ed. Iu.R. SheliagSosonko. In: Roslynnist' Ukrayiny, Kyiv: Phytosociocentre, 2006, 412 р. [Дубина Д.В. Вища водна рослинність / Відпов. ред. Ю.Р. Шеляг-Сосонко // Рослинність України. - К.: Фітосоціоцентр, 2006. - 412 с.].
Felzines J.-C. Contribution au prodromme des végétations de France: les Lemnetea minoris Tüxen ex O. Bòlos \& Masclans 1955, J. Bot. Soc. Bot. France, 2012, 59, pp. 189-240.

Hennekens S.M., Schaminée J.H.J. TURBOVEG, a comprehensive database management system for vegetation data, J. Vegetation Sci., 2001, 12, pp. 589-591.

Hrivnák R. Aquatic plant communities in the catchment area of the Ipel' river in Slovakia and Hungary. Part I. Classes Lemnetea and Charetea fragilis, Thaiszia, J. Bot, 2002, 12, pp. 25-50.

Husák Š. Utricularia L. - bublinatka. In: Květena České republiky. Ed. B. Slavik, vol. 6, Praha: Academia, 2000, S. 517-528.

Iakushenko D., Borysova O. Plant communities of the class Charetea Fukarek ex Krausch 1964 in Ukraine: an overview, Biodiv. Res. Conserv., 2012, 27, pp. 75-82.

Iakushenko D.M., Iusyp S.V., Solomakha V.A., Chorney I.I., Tokariuk A.I., Budzhak V.V., Danylyk I.M., Tomich M.V., Solomakha I.V., Derzhypilskyi L.M., Senchina B.V., Roslynnist' NPP «Gutsulshchina». In: Natsionalnyi pryrodnyi park «Gutsulshchina». Roslynnyi svit. Pryrodno-zapovidni terytorii Ukrayiny. Roslynnyi svit, Kyiv: Phytosociocentre, 2011, Vyp. 9, pp. 194-298 [Якушенко Д.М., Юсип С.В., Соломаха В.А., Чорней I.I., Токарюк А.I., Буджак В.В., Данилик I.M., Томич М.В., Соломаха I.В., Держипільський Л.М., Сенчина Б.В. Рослинність НПП «Гуцульщина»/ Національний природний парк «Гуиульщина». Рослинний світ. - Природно-заповідні території України. Рослинний світ. - Вип. 9. - К.: Фітосоціоцентр, 2011. - C. 194-298].

Lastrucci L., Bonari G., Angiolini C., Casini F., Giallonardo T., Gigante D., Landi M., Landucci F., Venanzoni R., Viciaini D. Vegetation of Lakes Chuisi and Montepulciano (Siena, central Italy): updated knowledge and new discoveries, Plant Sociology, 2014, 52(2), pp. 29-55.

Lawesson J.E. A tentative annotated checklist of Danish syntaxa, Folia Geobotanica, 2004, 39, pp. 73-95.

Müller T.H., Görs S. Pflanzengesellschaften stehender ewässer in Baden-Württenberg, Beitr. Z. Naturk. Forschung i. Südwestdeutchland, 1960, 19(1), pp. 60-100.

Pott R. Die Pflanzengesellschaften Deutschland, Stuttgart: E. Ulmer Verlag, 1995, 622 S.

Prots' B.G. Utricularia australis. In: Red Data Book of Ukraine. Vegetable Kingdom. Ed. Ia.P. Didukh, Kyiv: Globalkonsaltyng, 2009, p. 513 [Проць Б.Г. Пухирник південний - Utricularia australis // Червона книга України. Рослинний світ / За ред. Я.П. Дідуха. - К.: Глобалконсалтинг, 2009. - С. 513].

Rivas-Martínez S., Fernández-González F., Loidi J., Lousã M., Penas A. Syntaxonomical checklist of vascular plant communities of Spain and Portugal to association level, Itinera Geobotanica, 2001, 14, pp. 5-341.

Schratt L. Lemnetea, Die Pflanzengesellschaften Österreichs. 2. Natürliche waldfreie Vegetation, Jena-StuttgartNew York: G. Fischer Verlag, 1993, pp. 31-44. 
Sinkevičienè Z. Confirmation of Utricularia australis occurrence in Lithuania, Botanica Lithuanica, 2013, 19(2), pp. 161-164.

Solomakha V.A. Syntaksonomia roslynnosti Ukrayiny. Trete nablyzhennia, Kyiv: Phytosociocentre, 2008, 296 p. [Coломаха В.А. Синтаксономія рослинності України. Третє наближення. - К.: Фітосоціоцентр, 2008. - 296 с.].

Spałek K. Utricularietum australis Th. Müller et Görs 1960 in Poland, Acta Societatis Botanicorum Poloniae, 2006, 75(3), pp. 253-256.

Šumberová K. Vegetation of the Czech Republic. Ed. M. Chytrý. Vol. 3. Aquatic and wetland vegetation (Lemnetea), Praha: Academia, 2011, pp. 43-99.

Šumberová K., Hájková P., Chytrý M., Hroudová Z., Sádlo J., Hájek M., Hrivnák R., Navritolová J., Hanáková P., Ekrt L., Ekrtová E. Vegetation of the Czech Republic. Ed. M. Chytrý. Vol. 3. Aquatic and wetland vegetation (Phragmito-Magno-Caricetea), Praha: Academia, 2011, pp. 385-579.

Taylor P. Utricularia L. In: Flora Europaea. Ed. T.G. Tutin, Cambridge, 1972, vol. 3. pp. 296-297.

Tichý L. JUICE, software for vegetation classification, J. Vegetation Sci., 2002, 13, pp. 451-453.

Uotila P. Lentibulariaceae. In: Euro+Med Plantbase - the information resource for Euro-Mediterranean plant diversity, available at: http:// ww 2. bg b m . org / Euro Plus M ed/P Tax on Detail.asp? Name Cache $=$ Utricularia $\% 20$ australis\&PTRefFk=7500000 (accessed 4.02.2015).

Visiulina O.D. Utricularia. In: Flora URSR, Kyiv: AN URSR, 1961, vol. 10, pp. 61-67 [Вісюліна О.Д. Рід Пухирник - Utricularia L. // Флора УРСР. - Т. 10. - К.: Вид-во АН УРСР, 1961. - С. 61-67].

Westhoff V., van der Maarel E. The Braun-Blanquet approach. In: Classification of Plant Communities. Ed. R. Whittaker, The Hague: Junk, 1973, pp. 287-399.

Żukowski W. Rozmieszczenie gatunków $\mathrm{Z}$ rodzaju Utricularia L. w Polsce, Badania fizjograficzne nad Polska Zachodnia, Ser. B, Botanika, 1974, 28, S. 189-217.

Żukowski W. Utricularia L. Pływacz. In: Flora Polska, Warszawa;Kraków, 1970, vol. 11, S. 56-68.

Recommended for publication by

Submitted 03.08.2015.

S.L. Mosyakin
Якушенко Д.М. ${ }^{1}$, Орлов О.О. ${ }^{2}$. Нові знахідки Utricularia australis (Lentibulariaceae) в Україні. - Укр. ботан. журн. 2015. - 72(5): 468-473.

13еленогурський університет, факультет біологічних наук

вул. 3. Шафрана, 1, м. Зелена Гура, 65-516, Польща d.iakushenko@wnb.uz.zgora.pl

${ }^{2}$ Поліський філіал УкрНДІЛГА імені Г.М. Висоцького вул. Нескорених, 2, с. Довжик, Житомирський р-н, Житомирська обл., 10004, Україна

polysskiy_branch@ukr.net

У статті наводяться відомості про перші знахідки Utricularia australis на Українському Поліссі (Житомирська область). Подано екологічну та ценотичну характеристики виду в нових місцезнаходженнях. Локалітети U. australis представлені невеликими штучними водоймами в глиняних, торфових і гранітних кар'єрах, а також ставками. Виявлено, що Utricularia australis зростає в чотирьох флористичних асоціаціях: Utricularietum australis Müller et Görs 1960, Nitelletum mucronatae Corillion et Guerlesquin 1972, Equiseto fluviatilis-Caricetum rostratae Zumpfe 1929, Equisetetum fluviatilis Nowiński 1930. Уперше для територіï України вказано асоціацію Utricularietum australis і наведено їі характеристику.

К л ю ч о в і с л о в а: Utricularia australis, водна рослинність, флора, Україна.

Якушенко Д.Н. ${ }^{1}$, Орлов А.А. ${ }^{2}$. Новые находки Utricularia australis (Lentibulariaceae) в Украине. - Укр. ботан. журн. - 2015. - 72(5): 468-473.

13еленогурский университет, факультет биологических наук

ул. 3. Шафрана, 1, г. Зелёна Гура, 65-516, Польша

d.iakushenko@wnb.uz.zgora.pl

${ }^{2}$ Полесский филиал УкрНИИЛХА имени

Г.Н. Высоцкого

ул. Нескоренных, 2, с. Довжик, Житомирский р-н, Житомирская обл., 10004, Украина

polysskiy_branch@ukr.net

В статье приводятся данные о первых находках Utricularia australis в Украинском Полесье (Житомирская область), экологическая и ценотическая характеристики вида в новых местонахождениях. Локалитеты U. australis представлены небольшими искусственными водоемами в глиняных, торфяных и гранитных карьерах, а также прудами. Выявлено, что Utricularia australis произрастает в четырех флористических ассоциациях: Utricularietum australis Müller et Görs 1960, Nitelletum mucronatae Corillion et Guerlesquin 1972, Equiseto fluviatilis-Caricetum rostratae Zumpfe 1929, Equisetetum fluviatilis Nowiński 1930. Впервые для территории Украины указана ассоциация Utricularietum australis и приведена её характеристика.

К л ю ч е в ы е с л о в а: Utricularia australis, водная растительность, флора, Украина. 